\title{
Synthesis of Derivatives of $6 H$-1,2-Oxazine by Cyclization of Ketoximes With Derivatives of Terminal Acetylene Compounds
}

\author{
Raad Kasim Yahya ${ }^{1}$, Narayana U. Kudva N. ${ }^{1} \&$ K. M. L. Rai ${ }^{1}$ \\ ${ }^{1}$ Department of Studies in Chemistry, University of Mysore, Manasagangotri, Mysore, Karnataka, India \\ Correspondence: Raad Kasim Yahya, Department of Studies in Chemistry, University of Mysore, \\ Manasagangotri, Mysore 570006, India. E-mail: raad_1965@yahoo.com
}

Received: August 14, $2013 \quad$ Accepted: September 22, $2013 \quad$ Online Published: December 16, 2013
$\begin{aligned} & \text { doi:10.5539/ijc.v6n1p26 } \\ & \text { URL: http://dx.doi.org/10.5539/ijc.v6n1p26 }\end{aligned}$

\begin{abstract}
A new series of $6 H$-1,2-oxazines were synthesized by the reaction of ketones with hydroxylamine hydrochloride in the presence of sodium acetate to give ketoximes 1(a-e). Chloramine-T was used as an effective reagent for the generation of $\alpha$-nitrosolefins from ketoximes $\mathbf{1}(\mathbf{a}-\mathbf{e})$ which subsequently underwent hetero Diels-Alder reaction with terminal acetylenes $\mathbf{4 ( \mathbf { a } - \mathbf { d } ) \text { , to give } 6 H - 1 , 2 - O x a z i n e ~ d e r i v a t i v e s ~} \mathbf{5}(\mathbf{a}-\mathbf{t})$.
\end{abstract}

Keywords: $6 H$-1,2-Oxazine, $\alpha$-nitrosolefins, terminal acetylene, ketoximes, cycloaddition

\section{Introduction}

A broad range of synthetic applications demonstrates that 1,2-oxazine derivatives constitute a versatile class of N,O heterocycles (Gilchrist, 1983; Tsoungas, 2002; Zimmer et al., 2009; Lyapkalo \& Ioffe, 1998; Tishkov et al., 2002; Young \& Kerr, 2003; Helms et al., 2005; Cardona \& Goti, 2005; Sibi et al., 2005; Kumarn et al., 2006; Lu \& Zakarian, 2008; Brasholz et al., 2009; Sukhorukov et al., 2009). Considerable attention has been paid to 6H-1,2-oxazines bearing a C-4, C-5-double bond (Homann et al., 1998; Zimmer et al., 2002; Zimmer \& Reissig, 1988; Zimmer \& Reissig, 1991; Zimmer \& Reissig, 1992) which are useful intermediates in the synthesis of $\gamma$-lactams (Zimmer et al., 1992), $\gamma$-aminoacids, aminoalcohols (Zimmer et al., 1992), aziridines (Zimmer et al., 1993), pyrrolizidines (Zimmer et al., 2008), pyrrolidine derivatives (Zimmer et al., 2002; Buchholz \& Reissig, 2003; Reissig et al., 2007), 2-acetylpyridines (Thomas et al., 1987) and 2-(aminoalkyl) benzoic acids (Gilchrist \& Wood, 1996).

$\alpha$-Nitrosolefins are usually generated in situ from an $\alpha$-haloketoxime, by reaction with a heterogenous base such as sodium carbonate in an organic solvent (dichloromethane, diethyl ether, or methyl $t$-butyl ether) at room temperature (David et al., 1983; Shigeo et al., 1991; Mori \& Wu, 1991). Sung et al. (2001) prepared 1-aryl-2,2-dihaloethanone oximes from 1-aryl-2,2-dihaloethanone and hydroxylamine hydrochloride and then treated with sodium carbonate in dried ether. Manjula et al. (2009) used chloramine-T for the generation $\alpha$ nitrosolefins from ketoximes bearing $\alpha$-Hydrogen.

$6 H$-1,2-Oxazines were prepared from 1-bromo-2-ethoxyethene and $\alpha$-bromo-( $p$-methoxy) acetophenonoxime via $4+2$ cycloaddition followed by $\mathrm{HBr}$ elimination (Buchholz \& Reibig, 2003). An unusual synthesis of $6 H$-1,2-oxazines was accomplished by the reaction of nitrile oxides with vinylcarbene (Mark et al., 1993), which apparently involves 3-centre plus 3-centre cycloaddition of the dipole to the intermediate vinylcarbene. In this work we react various acetophenone oximes with chloramine-T and triethyl amine to afford $\alpha$-nitrosolefins which are subsequently reacted with terminal acetylenes to yield derivatives of $6 H$-1,2-oxazine.

\section{Experimental Section}

\subsection{General}

Analytical thin layer chromatography (TLC) was performed on $0.25 \mathrm{~mm}$ silica gel $60-\mathrm{F}$ plates and visualized by ultraviolet irradiation and $\mathrm{KMnO}_{4}$. Melting points were determined on Thomas Hoover melting point apparatus and are uncorrected. ${ }^{1} \mathrm{H}$ NMR spectra were recorded on a Bruker $\mathrm{AM}(300 \mathrm{MHz})$ spectrometer using $\mathrm{CDCl}_{3}$ as solvent and tetramethylsilane as internal standard. ${ }^{13} \mathrm{C}$ NMR spectra were measured on Jeol $400(300 \mathrm{MHz})$ instrument. Mass spectra obtained on a Finnigan 4021. Elemental analyses were carried out using Flash EA 1112 Series, CHN Analyzer (Thermo). IR spectra in $\mathrm{KBr}$ pellets were recorded on JASCO FT/IR-4100 FTIR spectrophotometer. 


\subsection{General Procedure for the Synthesis of Oxazine Derivatives 5(a-t)}

A mixture of ketoxime $(5 \mathrm{mmol})$ and chloramine-T trihydrate $(5.1 \mathrm{mmol})$ in ethanol $(20 \mathrm{ml})$ were refluxed for 4 hrs. The mixture was cooled to room temperature; triethylamine $(1 \mathrm{ml})$ was added and stirred at room temperature for $1 \mathrm{~h}$. After this, solution of terminal acetylene $(5.1 \mathrm{mmol})$ in ethanol $(5 \mathrm{ml})$ was added and stirred at reflux for 15-20 hours. It was then concentrated under reduced pressure and the residue is extracted with ether $(50 \mathrm{ml})$. This extract was then washed with water $(15 \mathrm{ml})$, aqueous $5 \% \mathrm{NaOH}(30 \mathrm{ml})$ and dried over anhydrous sodium sulfate. The solvent was evaporated and the remaining solid was recrystallized from $10 \%$ ethanol in $\mathrm{n}$-hexane to afford the pure compound.

\subsection{1 (3-Phenyl-6H-1,2-Oxazine-6-yl)-Methanol (5a)}

Obtained from 1a $(0.74 \mathrm{~g}, 5 \mathrm{mmol})$, chloramine- $\mathrm{T}_{3} 3 \mathrm{H}_{2} \mathrm{O}(1.43 \mathrm{~g}, 5.1 \mathrm{mmol})$ and $4 \mathrm{a}(0.28 \mathrm{~g}, 5.1 \mathrm{mmol})$ as a colorless solid, yield $(0.6 \mathrm{~g}, 64.5 \%)$, m.p. $89-90{ }^{\circ} \mathrm{C},{ }^{1} \mathrm{H}$ NMR $\left(\mathrm{CDCl}_{3}, 300 \mathrm{MHz}\right): \delta 2.13$ (br, $\left.1 \mathrm{H}, \mathrm{OH}\right), 3.75-3.93$ $\left(\mathrm{m}, 2 \mathrm{H}, \mathrm{CH}_{2}\right), 4.20(\mathrm{~m}, 1 \mathrm{H}, \mathrm{C} 6), 6.33(\mathrm{dd}, J=4.6 \mathrm{~Hz}, J=10.1 \mathrm{~Hz}, 1 \mathrm{H}, \mathrm{C} 5), 6.69$ (d, $\left.J=10.2 \mathrm{~Hz}, 1 \mathrm{H}, \mathrm{C} 4\right), 7.40$ $(\mathrm{t}, J=6 \mathrm{~Hz}, 2 \mathrm{H}, \mathrm{CH}), 7.66-7.69(\mathrm{~m}, 3 \mathrm{H}, \mathrm{CH}) .{ }^{13} \mathrm{C} \mathrm{NMR}\left(300 \mathrm{MHz}, \mathrm{CDCl}_{3}\right) ; 64.7,90.1,110.9,126.5,127.8$ 130.1, 132.3, 137.6, 156.2. IR (KBr pellets $\left.\mathrm{cm}^{-1}\right)$ v 1280, 1450, 1600, 1615, 2970, 3105, 3340. Anal. CHN: calcd C 69.83, H 5.86, N 7.40, found C 69.64, H 5.94, N 7.33. LC-MS: calcd for $\mathrm{C}_{11} \mathrm{H}_{11} \mathrm{NO}_{2}\left[\mathrm{M}^{+}\right]$: 189.08, found 189.90.

\subsection{2 (3-p-Tolyl-6H-1,2-Oxazine-6-yl)-Methanol (5b)}

Obtained from $\mathbf{1 b}(0.74 \mathrm{~g}, 5 \mathrm{mmol})$, chloramine- $\mathrm{T} .3 \mathrm{H}_{2} \mathrm{O}(1.43 \mathrm{~g}, 5.1 \mathrm{mmol})$ and $4 \mathbf{a}(0.28 \mathrm{~g}, 5.1 \mathrm{mmol})$ as a brown solid, yield $(0.6 \mathrm{~g}, 60 \%)$, m.p. $131-132{ }^{\circ} \mathrm{C},{ }^{1} \mathrm{H}$ NMR $\left(\mathrm{CDCl}_{3}, 300 \mathrm{MHz}\right): \delta 2.15$ (br, $\left.1 \mathrm{H}, \mathrm{OH}\right), 2.36(\mathrm{~s}, 3 \mathrm{H}$, $\left.\mathrm{CH}_{3}\right), 3.77-3.95\left(\mathrm{~m}, 2 \mathrm{H}, \mathrm{CH}_{2}\right), 4.22(\mathrm{~m}, 1 \mathrm{H}, \mathrm{C} 6), 6.28(\mathrm{dd}, J=4.6 \mathrm{~Hz}, J=10.1 \mathrm{~Hz}, 1 \mathrm{H}, \mathrm{C} 5), 6.70(\mathrm{~d}, J=10.2$, $1 \mathrm{H}, \mathrm{C} 4), 7.19$ (dd, $J=7 \mathrm{~Hz}, J=2 \mathrm{~Hz}, 2 \mathrm{H}, \mathrm{CH}), 7.60$ (dd, $J=6 \mathrm{~Hz}, J=2 \mathrm{~Hz}, 2 \mathrm{H}, \mathrm{CH}){ }^{13} \mathrm{C}$ NMR $(300 \mathrm{MHz}$, $\left.\mathrm{CDCl}_{3}\right) ; 23.3,64.3,90.2,110.6,125.2,127.7,133.2,140.8,156.3$. IR $\left(\mathrm{KBr}\right.$ pellets $\left.\mathrm{cm}^{-1}\right)$ v1280, 1445, 1600, 1612, 2980, 3110, 3350. Anal. CHN: calcd C 70.92, H 6.45, N 6.89, found C 70.99, H 6.35, N 6.99. LC-MS: calcd for $\mathrm{C}_{12} \mathrm{H}_{13} \mathrm{NO}_{2}\left[\mathrm{M}^{+}\right]$: 203.09, found 204.10.

\subsection{3 (3-(4-Chlorophenyl)-6H-1,3-Oxazin-6-yl)-Methanol (5c)}

Obtained from 1c $(0.84 \mathrm{~g}, 5 \mathrm{mmol})$, chloramine- $\mathrm{T}^{2} 3 \mathrm{H}_{2} \mathrm{O}(1.43 \mathrm{~g}, 5.1 \mathrm{mmol})$ and $4 \mathrm{a}(0.28 \mathrm{~g}, 5.1 \mathrm{mmol})$ as a brown oil, yield $(0.6 \mathrm{~g}, 54 \%),{ }^{1} \mathrm{H}$ NMR $\left(\mathrm{CDCl}_{3}, 300 \mathrm{MHz}\right): \delta 2.16(\mathrm{br}, 1 \mathrm{H}, \mathrm{OH}), 3.76-3.90\left(\mathrm{~m}, 2 \mathrm{H}, \mathrm{CH}_{2}\right), 4.23$ (m, 1H, OCH), 6.25 (dd, $J=4.6 \mathrm{~Hz}, J=10.1 \mathrm{~Hz}, 1 \mathrm{H}, \mathrm{C} 5), 6.32(\mathrm{~d}, J=10.1 \mathrm{~Hz}, 1 \mathrm{H}, \mathrm{C} 4), 7.44$ (d, $J=8.7 \mathrm{~Hz}, 2 \mathrm{H}$, $\mathrm{CH}), 7.68(\mathrm{~d}, J=8.7 \mathrm{~Hz}, 2 \mathrm{H}, \mathrm{CH}){ }^{13} \mathrm{C}$ NMR $\left(300 \mathrm{MHz}, \mathrm{CDCl}_{3}\right) ; 65.6,90.3,110.6,125.5,127.9,129.8,133.3$, 139.8, 156.3. IR (neat, $\mathrm{cm}^{-1}$ ) v1285, 1440, 1600, 1610, 2960, 3100, 3360. Anal. CHN: calcd C 59.07, H 4.51, N 6.26, found C 59.17, H.4.41, N6.36. LC-MS: calcd for $\mathrm{C}_{11} \mathrm{H}_{10} \mathrm{ClNO}_{2}\left[\mathrm{M}^{+}\right]$: 223.04, found 225.05.

\subsection{4 (3-(4-Methoxyphenyl)-6H-yl)-Methanol (5d)}

Obtained from 1d $(0.82 \mathrm{~g}, 5 \mathrm{mmol})$, chloramine-T. $3 \mathrm{H}_{2} \mathrm{O}(1.43 \mathrm{~g}, 5.1 \mathrm{mmol})$ and $4 \mathrm{a}(0.28 \mathrm{~g}, 5.1 \mathrm{mmol})$ as a brown oil, yield $(0.7 \mathrm{~g}, 64.8 \%),{ }^{1} \mathrm{H}$ NMR $\left(\mathrm{CDCl}_{3}, 300 \mathrm{MHz}\right): \delta 2.14(\mathrm{br}, 1 \mathrm{H}, \mathrm{OH}), 3.77-3.95\left(\mathrm{~m}, 5 \mathrm{H}, \mathrm{CH}_{2}\right.$, $\left.\mathrm{OCH}_{3}\right), 4.24(\mathrm{~m}, 1 \mathrm{H}, \mathrm{C} 6), 6.28$ (dd, $\left.J=4.6 \mathrm{~Hz}, J=10.1 \mathrm{~Hz}, 1 \mathrm{H}, \mathrm{C} 5\right), 6.68(\mathrm{~d}, J=10.2 \mathrm{~Hz}, 1 \mathrm{H}, \mathrm{C} 4), 7.19(\mathrm{dd}, J=$ $7 \mathrm{~Hz}, J=2 \mathrm{~Hz}, 2 \mathrm{H}, \mathrm{CH}), 7.60(\mathrm{dd}, J=6 \mathrm{~Hz}, J=2 \mathrm{~Hz}, 2 \mathrm{H}, \mathrm{CH}){ }^{13} \mathrm{C}$ NMR $\left(300 \mathrm{MHz}, \mathrm{CDCl}_{3}\right) ; \quad 58.8,65.2,90.6$, 110.9, 118.8, 126.1, 127.7, 134.4, 156.3, 166.7. IR (neat, $\mathrm{cm}^{-1}$ ) v1247, 11290, 1442, 1618, 2950, 3000, 3030, 3070, 3360. Anal. CHN: calcd C 65.74, H 5.98, N 6.39, found C 65.80, H 5.88, N6.50. LC-MS: calcd for $\mathrm{C}_{12} \mathrm{H}_{13} \mathrm{NO}_{3}\left[\mathrm{M}^{+}\right]: 219.09$, found .219 .93 .

\subsection{5 (3-(4-Nitrophenyl)-6H-1,2-Oxazin-6-yl)-Methanol (5e)}

Obtained from 1e $(0.9 \mathrm{~g}, 5 \mathrm{mmol})$, chloramine- $\mathrm{T} .3 \mathrm{H}_{2} \mathrm{O}(1.43 \mathrm{~g}, 5.1 \mathrm{mmol})$ and $4 \mathbf{a}(0.28 \mathrm{~g}, 5.1 \mathrm{mmol})$ as a colorless solid, yield $(0.7 \mathrm{~g}, 60 \%)$, m.p. $131-132{ }^{\circ} \mathrm{C},{ }^{1} \mathrm{H}$ NMR $\left(\mathrm{CDCl}_{3}, 300 \mathrm{MHz}\right): \delta 2.16(\mathrm{br}, 1 \mathrm{H}, \mathrm{OH})$, 3.70-3.90 (m, 2H, CH $), 4.21$ (m, 1H, C6), 6.26 (dd, $J=4.6, J=10.1 \mathrm{~Hz}, 1 \mathrm{H}, \mathrm{C} 5), 6.71$ (d, $J=10.2 \mathrm{~Hz}, 1 \mathrm{H}, \mathrm{C} 4)$, $8.30(\mathrm{~d}, J=8.9 \mathrm{~Hz}, 2 \mathrm{H}, \mathrm{CH}), 8.34(\mathrm{~d}, J=8.9 \mathrm{~Hz}, 2 \mathrm{H}, \mathrm{CH}){ }^{13} \mathrm{C}$ NMR $\left(300 \mathrm{MHz}, \mathrm{CDCl}_{3}\right) ; 65.3,90.7,110.5$, 125.2, 127.9, 134.5, 136.7, 154.3, 156.3. IR (KBr pellets $\left.\mathrm{cm}^{-1}\right) v 1125,1435,1520,1615,2980,3030,3100,3365$. Anal. CHN: calcd C 56.41, H 4.30, N 11.96, found C 56.56, H 4.20, N 11.90. LC-MS: calcd for $\mathrm{C}_{11} \mathrm{H}_{10} \mathrm{~N}_{2} \mathrm{O}_{4}$ $\left[\mathrm{M}^{+}\right]$: 234.06, found 235.25.

\subsubsection{6-Phenyl-3-p-Tolyl-6H-1,2-Oxazine (5f)}

Obtained from 1a $(0.67 \mathrm{~g}, 5 \mathrm{mmol})$, chloramine- $\mathrm{T}^{3} 3 \mathrm{H}_{2} \mathrm{O}(1.43 \mathrm{~g}, 5.1 \mathrm{mmol})$ and $\mathbf{4 b}(0.52 \mathrm{~g}, 5.1 \mathrm{mmol})$ as pale brown oil, yield (0.6 g, 51.7\%), ${ }^{1} \mathrm{H}$ NMR $\left(\mathrm{CDCl}_{3}, 300 \mathrm{MHz}\right): \delta 5.32(\mathrm{~m}, 1 \mathrm{H}, \mathrm{C} 6), 6.27$ (dd, $J=4.6 \mathrm{~Hz}, J=10.1$ $\mathrm{Hz}, 1 \mathrm{H}, \mathrm{C} 5), 6.72$ (d, $J=10.2 \mathrm{~Hz}, 1 \mathrm{H}, \mathrm{C} 4), 7.12$ (dd, J = 6 Hz, $J=2 \mathrm{~Hz}, 2 \mathrm{H}, \mathrm{CH}), 7.20-7.22(\mathrm{~m}, 5 \mathrm{H}, \mathrm{CH}), 7.40$ $(\mathrm{t}, J=6 \mathrm{~Hz}, 2 \mathrm{H}, \mathrm{CH}), 7.70-7.74(\mathrm{~m}, 3 \mathrm{H}, \mathrm{CH}) .{ }^{13} \mathrm{C} \mathrm{NMR}\left(300 \mathrm{MHz}, \mathrm{CDCl}_{3}\right) ; 90.4,110.8,127.9,128.2,129.1$, 131.1, 133.2, 135.1, 136.8, 138.1, 156.4. IR (neat, $\mathrm{cm}^{-1}$ ) v1600, 1610, 2980, 3108. Anal. CHN: calcd C 81.68, H 
5.57, N 5.95, found C 81.77, H 5.44, N 6.05. LC-MS: calcd for $\mathrm{C}_{17} \mathrm{H}_{15} \mathrm{NO}\left[\mathrm{M}^{+}\right]$: 249.12, found 249.95 .

\subsubsection{3,6-Diphenyl-6H-1,2-Oxazine (5g)}

Obtained from $\mathbf{1 b}(0.74 \mathrm{~g}, 5 \mathrm{mmol})$, chloramine- $\mathrm{T} .3 \mathrm{H}_{2} \mathrm{O}(1.43 \mathrm{~g}, 5.1 \mathrm{mmol})$ and $\mathbf{4 b}(0.52 \mathrm{~g}, 5.1 \mathrm{mmol})$ as colorless solid, yield $(0.7 \mathrm{~g}, 56.9 \%)$, m.p. $212-214{ }^{\circ} \mathrm{C},{ }^{1} \mathrm{H}$ NMR $\left(\mathrm{CDCl}_{3}, 300 \mathrm{MHz}\right): \delta 2.38\left(\mathrm{~s}, 3 \mathrm{H}, \mathrm{CH}_{3}\right), 5.33(\mathrm{~m}$, $1 \mathrm{H}, \mathrm{C} 6), 6.70(\mathrm{~d}, J=10.2 \mathrm{~Hz}, 1 \mathrm{H}, \mathrm{C} 4), 6.30(\mathrm{dd}, J=4.6 \mathrm{~Hz}, J=10.1,1 \mathrm{H}, \mathrm{C} 5), 7.30(\mathrm{t}, J=6.1 \mathrm{~Hz}, 2 \mathrm{H}, \mathrm{CH})$, 7.65-7.68 (m, 3H, CH), 8.33 (d, $J=8.9 \mathrm{~Hz}, 2 \mathrm{H}, \mathrm{CH}), 8.37(\mathrm{~d}, J=8.9 \mathrm{~Hz}, 2 \mathrm{H}, \mathrm{CH}),{ }^{13} \mathrm{C}$ NMR $\left(300 \mathrm{MHz}, \mathrm{CDCl}_{3}\right)$; 24.4, 90.5, 110.9, 125.4, 127.9, 129.3, 131.2, 133.2, 135.5, 141.2, 156.4. IR (neat, $\mathrm{cm}^{-1}$ ) v1370, 1590, 1620, 2980 , 3080. Anal. CHN: calcd C 81.90, H 6.06, N 5.62, found C 81.80, H 6.24, N 4.51. LC-MS: calcd for $\mathrm{C}_{16} \mathrm{H}_{13} \mathrm{NO}$ $\left[\mathrm{M}^{+}\right]$: 235.10, found 235.95 .

\subsubsection{3-(4-Chlorophenyl)-6-Phenyl-6H-1,2-Oxazine (5h)}

Obtained from $1 \mathrm{c}(0.84 \mathrm{~g}, 5 \mathrm{mmol})$, chloramine- $\mathrm{T}^{2} \mathrm{H}_{2} \mathrm{O}(1.43 \mathrm{~g}, 5.1 \mathrm{mmol})$ and $\mathbf{4 b}(0.52 \mathrm{~g}, 5.1 \mathrm{mmol})$ as a colorless solid, yield $(0.7 \mathrm{~g}, 52.6 \%)$, m.p. $131-132^{\circ} \mathrm{C},{ }^{1} \mathrm{H}$ NMR $\left(\mathrm{CDCl}_{3}, 300 \mathrm{MHz}\right): \delta 5.35(\mathrm{~m}, 1 \mathrm{H}, \mathrm{C} 6), 6.71$ ( d, $J=10.2 \mathrm{~Hz}, 1 \mathrm{H}, \mathrm{C} 4), 6.31(\mathrm{dd}, J=4.6 \mathrm{~Hz}, J=10.1 \mathrm{~Hz}, 1 \mathrm{H}, \mathrm{C} 5), 7.30(\mathrm{t}, J=6 \mathrm{~Hz}, 2 \mathrm{H}, \mathrm{CH}), 7.42(\mathrm{~d}, J=8.7 \mathrm{~Hz}$, 2H), $7.63(\mathrm{~d}, J=8.7 \mathrm{~Hz}, 2 \mathrm{H}, \mathrm{CH}), 7.75-7.78(\mathrm{~m}, 3 \mathrm{H}, \mathrm{CH}) .{ }^{13} \mathrm{C}$ NMR $\left(300 \mathrm{MHz}, \mathrm{CDCl}_{3}\right) ; 90.4,110.7,126.6$, 127.9 128.9, 129.9, 133.2, 135.5, 138.9, 146.4, 156.3. IR $\left(\mathrm{KBr}\right.$ pellets $\left.\mathrm{cm}^{-1}\right)$ v1580, 1615, 2965, 3100. Anal. CHN: calcd C 71.25, H 4.48, N 5.19, found C 71.40, H 4.34, N 5.40. LC-MS: calcd for $\mathrm{C}_{16} \mathrm{H}_{12} \mathrm{ClNO}\left[\mathrm{M}^{+}\right.$]: 269.06, found 271.05.

\subsubsection{3-(4-Methoxyphenyl)-6-Phenyl-6H-1,2-Oxazine (5i)}

Obtained from $1 \mathbf{d}(0.82 \mathrm{~g}, 5 \mathrm{mmol})$, chloramine-T. $3 \mathrm{H}_{2} \mathrm{O}(1.43 \mathrm{~g}, 5.1 \mathrm{mmol})$ and $\mathbf{4 b}(0.52 \mathrm{~g}, 5.1 \mathrm{mmol})$ as a brown oil, yield $(0.8 \mathrm{~g}, 61 \%),{ }^{1} \mathrm{H}$ NMR $\left(\mathrm{CDCl}_{3}, 300 \mathrm{MHz}\right): \delta 3.77\left(\mathrm{~s}, 3 \mathrm{H}, \mathrm{OCH}_{3}\right), 5.30(\mathrm{~m}, 1 \mathrm{H}, \mathrm{OCH}), 6.72(\mathrm{~d}, J$ $=10.2 \mathrm{~Hz}, 1 \mathrm{H}, \mathrm{C} 4), 6.28(\mathrm{dd}, J=4.6 \mathrm{~Hz}, J=10.1 \mathrm{~Hz}, 1 \mathrm{H}, \mathrm{C} 5), 6.90(\mathrm{dd}, J=7 \mathrm{~Hz}, J=2 \mathrm{~Hz}, 2 \mathrm{H}, \mathrm{CH}), 7.33(\mathrm{t}, J$ $=6 \mathrm{~Hz}, 2 \mathrm{H}, \mathrm{CH}), 7.55-7.65(\mathrm{~m}, 3 \mathrm{H}, \mathrm{CH}), 7.70(\mathrm{dd}, J=7 \mathrm{~Hz}, J=2 \mathrm{~Hz}, 2 \mathrm{H}, \mathrm{CH}),{ }^{13} \mathrm{C}$ NMR $\left(300 \mathrm{MHz}, \mathrm{CDCl}_{3}\right)$; $58.7,90.7,110.5,112.8,122.5,127.6,129.9,130.3,133.2,134.9,140.1,156.3,166.1$. IR (neat, $\mathrm{cm}^{-1}$ ) v1240, 1600, 1612, 2830, 2945, 3000, 3065. Anal. CHN: calcd C 76.96, H 5.70, N 5.28, found C 76.90, H 5.84, N 5.40. LC-MS: calcd for $\mathrm{C}_{17} \mathrm{H}_{15} \mathrm{NO}_{2}\left[\mathrm{M}^{+}\right]$: 265.11, found 265.95.

\subsubsection{3-(4-Nitrophenyl-6-Phenyl-6H-1,2-Oxazine (5j)}

Obtained from 1e $(0.9 \mathrm{~g}, 5 \mathrm{mmol})$, chloramine- $\mathrm{T}^{3} \mathrm{H}_{2} \mathrm{O}(1.43 \mathrm{~g}, 5.1 \mathrm{mmol})$ and $\mathbf{4 b}(0.52 \mathrm{~g}, 5.1 \mathrm{mmol})$ as a pale yellow oil, yield $(0.8 \mathrm{~g}, 57.1 \%),{ }^{1} \mathrm{H}$ NMR $\left(\mathrm{CDCl}_{3}, 300 \mathrm{MHz}\right): \delta 5.35(\mathrm{~m}, 1 \mathrm{H}, \mathrm{C} 6), 6.72(\mathrm{~d}, J=10.2 \mathrm{~Hz}, 1 \mathrm{H}, \mathrm{C} 4)$, $6.30(\mathrm{dd}, J=4.6 \mathrm{~Hz}, J=10.1 \mathrm{~Hz}, 1 \mathrm{H}, \mathrm{C} 5), 7.40(\mathrm{t}, J=6 \mathrm{~Hz}, 2 \mathrm{H}, \mathrm{CH}), 7.61-7.64(\mathrm{~m}, 3 \mathrm{H}, \mathrm{CH}), 7.90$ (d, 2H, $J=$ $8.8 \mathrm{~Hz}), 8.29$ (d, $2 \mathrm{H}, J=8.8 \mathrm{~Hz}){ }^{13} \mathrm{C}$ NMR $\left(300 \mathrm{MHz}, \mathrm{CDCl}_{3}\right)$; 90.7, 110.7, 125.5, 127.8, 128.9, 130.6, 132.3, 135.5, 138.7, 146.6, 152.6, 156.4. IR (neat, $\mathrm{cm}^{-1}$ ) v1345, 1520, 1580, 1610, 3075, 3110. Anal. CHN: calcd C 68.56, H 4.32, N 9.99, found C 68.67, $\mathrm{H} 4.24, \mathrm{~N}$ 9.80. LC-MS: calcd for $\mathrm{C}_{16} \mathrm{H}_{12} \mathrm{~N}_{2} \mathrm{O}_{3}\left[\mathrm{M}^{+}\right]$: 280.08, found 280.93 .

\subsubsection{Ethyl-3-Phenyl-6H-1,2-Oxazine-6-Carboxylate (5k)}

Obtained from 1a $(0.67 \mathrm{~g}, 5 \mathrm{mmol})$, chloramine- $\mathrm{T} .3 \mathrm{H}_{2} \mathrm{O}(1.43 \mathrm{~g}, 5.1 \mathrm{mmol})$ and $4 \mathbf{c}(0.5 \mathrm{~g}, 5.1 \mathrm{mmol})$ as a colorless solid, yield $(0.6 \mathrm{~g}, 52.6 \%)$, m.p. $131-132{ }^{\circ} \mathrm{C},{ }^{1} \mathrm{H}$ NMR $\left(\mathrm{CDCl}_{3}, 300 \mathrm{MHz}\right): \delta 1.38(\mathrm{t}, J=7.2 \mathrm{~Hz}, 3 \mathrm{H}$, $\left.-\mathrm{CO}_{2}-\mathrm{CH}_{2}-\mathrm{CH}_{3}\right), 4.30\left(\mathrm{q}, J=7.2 \mathrm{~Hz}, 2 \mathrm{H}, \mathrm{OCH}_{2}\right), 5.10(\mathrm{~d}, J=4.5 \mathrm{~Hz}, 1 \mathrm{H}, \mathrm{C} 6), 6.26$ (dd, $J=4.6 \mathrm{~Hz}, J=10.1$, $1 \mathrm{H}, \mathrm{C}$ ), 6.69 (d, $J=10.2 \mathrm{~Hz}, 1 \mathrm{H}, \mathrm{C} 4), 7.33(\mathrm{t}, J=6 \mathrm{~Hz}, 2 \mathrm{H}, \mathrm{CH}), 7.64-7.67(\mathrm{~m}, 3 \mathrm{H}, \mathrm{CH}) .{ }^{13} \mathrm{C}$ NMR $(300 \mathrm{MHz}$, $\left.\mathrm{CDCl}_{3}\right) ; 16.6,65.5,93.7,110.6,125.6,127.9,129.9,131.3,135.5,156.5,175.4$. IR $\left(\mathrm{KBr}\right.$ pellets cm $\left.{ }^{-1}\right) \vee 1375$, 1440, 1600, 1615, 1745, 2975, 3100. Anal. CHN: calcd C 67.52, H 5.67, N 6.06, found C 67.59, H 5.54, N 6.20. LC-MS: calcd for $\mathrm{C}_{13} \mathrm{H}_{13} \mathrm{NO}_{3}\left[\mathrm{M}^{+}\right]$: 231.09, found 232.05.

2.2.12 Ethyl-3-p-Tolyl-6H-1,2-Oxazine-6-Carboxylate (51)

Obtained from $\mathbf{1 b}(0.74 \mathrm{~g}, 5 \mathrm{mmol})$, chloramine- $\mathrm{T}^{2} 3 \mathrm{H}_{2} \mathrm{O}(1.43 \mathrm{~g}, 5.1 \mathrm{mmol})$ and $\mathbf{4 c}(0.5 \mathrm{~g}, 5.1 \mathrm{mmol})$ as brown solid, yield $(0.7 \mathrm{~g}, 57.8 \%)$, m.p. $131-132{ }^{\circ} \mathrm{C},{ }^{1} \mathrm{H}$ NMR $\left(\mathrm{CDCl}_{3}, 300 \mathrm{MHz}\right): \delta 1.40(\mathrm{t}, J=7.2 \mathrm{~Hz}, 3 \mathrm{H}$, $-\mathrm{CO}_{2}-\mathrm{CH}_{2}-\mathrm{CH}_{3}$ ), $2.36\left(\mathrm{~s}, 3 \mathrm{H}, \mathrm{CH}_{3}\right), 4.42$ (q, $J=7.2 \mathrm{~Hz}, 2 \mathrm{H}, \mathrm{OCH}_{2}$ ), 5.12 (d, $\left.J=4.5 \mathrm{~Hz}, 1 \mathrm{H}, \mathrm{C} 6\right), 6.29$ (dd, $J=$ 4.6, $J=10.1 \mathrm{~Hz}, 1 \mathrm{H}, \mathrm{C} 5), 6.68(\mathrm{~d}, J=10.2,1 \mathrm{H}, \mathrm{C} 4), 7.20(\mathrm{dd}, J=6 \mathrm{~Hz}, J=2 \mathrm{~Hz}, 2 \mathrm{H}, \mathrm{CH}), 7.60$ (dd, $J=6 \mathrm{~Hz}, J$ $=2 \mathrm{~Hz}, 2 \mathrm{H}, \mathrm{CH}) .{ }^{13} \mathrm{C} \mathrm{NMR}\left(300 \mathrm{MHz}, \mathrm{CDCl}_{3}\right) ; 17.7,24.4,65.5,93.9,110.7,127.9,128.8,133.3,141.9,156.4$, 175.5. IR $\left(\mathrm{KBr}\right.$ pellets $\left.\mathrm{cm}^{-1}\right) \vee 1370,1445,1580,1610,1740,2980,3110$. Anal. CHN: calcd C 68.56, H 6.16, N 5.71, found C 68.70, $\mathrm{H}$ 6.04, N 5.76. LC-MS: calcd for $\mathrm{C}_{14} \mathrm{H}_{15} \mathrm{NO}_{3}\left[\mathrm{M}^{+}\right]$: 245.11, found 246.04.

\subsubsection{Ethyl-3-(4-Chlorophenyl)-6H-1,2-Oxazine-6-Carboxylate (5m)}

Obtained from $1 \mathrm{c}(0.84 \mathrm{~g}, 5 \mathrm{mmol})$, chloramine- $\mathrm{T} .3 \mathrm{H}_{2} \mathrm{O}(1.43 \mathrm{~g}, 5.1 \mathrm{mmol})$ and $\mathbf{4 c}(0.5 \mathrm{~g}, 5.1 \mathrm{mmol})$ as a brown solid, yield $(0.8 \mathrm{~g}, 61 \%)$, m.p. $131-132{ }^{\circ} \mathrm{C},{ }^{1} \mathrm{H}$ NMR $\left(\mathrm{CDCl}_{3}, 300 \mathrm{MHz}\right): \delta 1.39$ (t, $J=7.2 \mathrm{~Hz}, 3 \mathrm{H}$, 
$\left.-\mathrm{CO}_{2}-\mathrm{CH}_{2}-\mathrm{CH}_{3}\right), 4.38\left(\mathrm{q}, J=7.2 \mathrm{~Hz}, 2 \mathrm{H}, \mathrm{OCH}_{2}\right), 5.11(\mathrm{~d}, J=4.5 \mathrm{~Hz}, 1 \mathrm{H}, \mathrm{C} 6), 6.26(\mathrm{dd}, J=4.6 \mathrm{~Hz}, J=10.1 \mathrm{~Hz}$, $1 \mathrm{H}, \mathrm{C} 5), 6.70(\mathrm{~d}, J=10.2 \mathrm{~Hz}, 1 \mathrm{H}, \mathrm{C} 4), 7.35(\mathrm{~d}, J=8.8 \mathrm{~Hz}, 2 \mathrm{H}, \mathrm{CH}), 7.67(\mathrm{~d}, J=8.8 \mathrm{~Hz}, 2 \mathrm{H}, \mathrm{CH}) .{ }^{13} \mathrm{C} \mathrm{NMR}$ $\left(300 \mathrm{MHz}, \mathrm{CDCl}_{3}\right) ; 17.7,65.5,93.7,110.6,127.8,129.9,133.2,135.5,139.9,156.3,176.6$. IR $\left(\mathrm{KBr}\right.$ pellets cm $\left.{ }^{-1}\right)$ v 1375, 1440, 1590, 1615, 1745, 2975, 3120. Anal. CHN: calcd C 58.77, H 4.55, N 5.27, found C 58.55, H 4.74, N 5.13. LC-MS: calcd for $\mathrm{C}_{13} \mathrm{H}_{12} \mathrm{ClNO}_{3}\left[\mathrm{M}^{+}\right]$: 265.05, found 267.03.

2.2.14 Ethyl-3-(4-Methoxyphenyl)-6H-1,2-Oxazine-6-Carboxylate (5n)

Obtained from 1d $(0.82 \mathrm{~g}, 5 \mathrm{mmol})$, chloramine- $\mathrm{T} .3 \mathrm{H}_{2} \mathrm{O}(1.43 \mathrm{~g}, 5.1 \mathrm{mmol})$ and $4 \mathbf{c}(0.5 \mathrm{~g}, 5.1 \mathrm{mmol})$ as a colorless solid, yield $(0.7 \mathrm{~g}, 54.2 \%)$, m.p. $131-132{ }^{\circ} \mathrm{C},{ }^{1} \mathrm{H}$ NMR $\left(\mathrm{CDCl}_{3}, 300 \mathrm{MHz}\right): \delta 1.37$ (t, $J=7.2 \mathrm{~Hz}, 3 \mathrm{H}$, $\left.-\mathrm{CO}_{2}-\mathrm{CH}_{2}-\mathrm{CH}_{3}\right), 3.82\left(\mathrm{~s}, 3 \mathrm{H}, \mathrm{OCH}_{3}\right), 4.40\left(\mathrm{q}, J=7.2 \mathrm{~Hz}, 2 \mathrm{H}, \mathrm{OCH}_{2}\right), 5.14(\mathrm{~d}, J=4.5 \mathrm{~Hz}, 1 \mathrm{H}, \mathrm{C} 6), 6.31(\mathrm{dd}, J=$ $4.6 \mathrm{~Hz}, J=10.1 \mathrm{~Hz}, 1 \mathrm{H}, \mathrm{C} 5), 6.71(\mathrm{~d}, J=10.2 \mathrm{~Hz}, 1 \mathrm{H}, \mathrm{C} 4), 6.80(\mathrm{dd}, J=7 \mathrm{~Hz}, J=2 \mathrm{~Hz}, 2 \mathrm{H}, \mathrm{CH}), 7.60(\mathrm{dd}, J$ $=7 \mathrm{~Hz}, J=2 \mathrm{~Hz}, 2 \mathrm{H}, \mathrm{CH}) .{ }^{13} \mathrm{C}$ NMR $\left(300 \mathrm{MHz}, \mathrm{CDCl}_{3}\right) ; 17.7,59.9,63.4,93.9,110.9,112.2,124.5,127.9,132.2$, 156.4 162.5, 176.7. IR (KBr pellets cm$\left.{ }^{-1}\right) \vee 1370,1445,1595,1610,1750,2988,3095$. Anal. CHN: calcd C 64.36, H 5.79, N 5.36, found C 64.50, H 5.54, N 5.40. LC-MS: calcd for $\mathrm{C}_{14} \mathrm{H}_{15} \mathrm{NO}_{4}\left[\mathrm{M}^{+}\right]: 260.10$, found 261.05.

\subsubsection{Ethyl-3-(4-Nitrophenyl)-6H-1,2-Oxazine-6-Carboxylalate (5o)}

Obtained from 1e $(0.9 \mathrm{~g}, 5 \mathrm{mmol})$, chloramine- $\mathrm{T} .3 \mathrm{H}_{2} \mathrm{O}(1.43 \mathrm{~g}, 5.1 \mathrm{mmol})$ and $4 \mathbf{c}(0.5 \mathrm{~g}, 5.1 \mathrm{mmol})$ as a pale yellow solid, yield $(0.8 \mathrm{~g}, 58.3 \%)$, m.p. $131-132{ }^{\circ} \mathrm{C},{ }^{1} \mathrm{H}$ NMR $\left(\mathrm{CDCl}_{3}, 300 \mathrm{MHz}\right): \delta 1.40(\mathrm{t}, J=7.2 \mathrm{~Hz}, 3 \mathrm{H}$, $\left.-\mathrm{CO}_{2}-\mathrm{CH}_{2}-\mathrm{CH}_{3}\right), 4.39$ (q, $\left.J=7.2 \mathrm{~Hz}, 2 \mathrm{H}, \mathrm{OCH}_{2}\right), 5.15(\mathrm{~d}, J=4.5 \mathrm{~Hz}, 1 \mathrm{H}, \mathrm{C} 6), 6.29$ (dd, $J=4.6, J=10.1 \mathrm{~Hz}, 1 \mathrm{H}$, C5), $6.73(\mathrm{~d}, J=10.2 \mathrm{~Hz}, 1 \mathrm{H}, \mathrm{C} 4), 8.30(\mathrm{~d}, J=8.9 \mathrm{~Hz}, 2 \mathrm{H}, \mathrm{CH}), 8.34(\mathrm{~d}, J=8.9 \mathrm{~Hz}, 2 \mathrm{H}, \mathrm{CH}){ }^{13} \mathrm{C}$ NMR (300 $\left.\mathrm{MHz}, \mathrm{CDCl}_{3}\right) ; 17.7,65.6,93.3,110.6,127.9,129.8,130.9,142.2,153.4,156.5,176.8$. IR $\left(\mathrm{KBr}\right.$ pellets cm $\left.{ }^{-1}\right) v$ 1370, 1440, 1525, 1595, 1615, 2990, 3070, 3110. Anal. CHN: calcd C 56.52, H 4.38, N 10.14, found C 56.40, H 4.54, N 10.04. LC-MS: calcd for $\mathrm{C}_{13} \mathrm{H}_{12} \mathrm{~N}_{2} \mathrm{O}_{5}\left[\mathrm{M}^{+}\right]$: 276.07, found 277.05 .

\subsubsection{3-Phenyl-6-Propyl-6H-1,2-Oxazine (5p)}

Obtained from 1a $(0.67 \mathrm{~g}, 5 \mathrm{mmol})$, chloramine- $\mathrm{T}^{2} 3 \mathrm{H}_{2} \mathrm{O}(1.43 \mathrm{~g}, 5.1 \mathrm{mmol})$ and $4 \mathbf{d}(0.35 \mathrm{~g}, 5.1 \mathrm{mmol})$ as a brown solid, yield $(0.6 \mathrm{~g}, 58.2 \%)$, m.p. $131-132{ }^{\circ} \mathrm{C},{ }^{1} \mathrm{H}$ NMR $\left(\mathrm{CDCl}_{3}, 300 \mathrm{MHz}\right): \delta 0.93\left(\mathrm{~s}, 3 \mathrm{H}, \mathrm{CH}_{3}\right), 1.37-1.42$ (m, 4H, $\left.\mathrm{CH}_{2}-\mathrm{CH}_{2}\right), 4.20(\mathrm{~m}, 1 \mathrm{H}, \mathrm{C} 6), 6.28$ (dd, $\left.J=4.6 \mathrm{~Hz}, J=10.1 \mathrm{~Hz}, 1 \mathrm{H}, \mathrm{C} 5\right), 6.68$ (d, $\left.J=10.2 \mathrm{~Hz}, 1 \mathrm{H}, \mathrm{C} 4\right)$, $7.39(\mathrm{t}, J=6 \mathrm{~Hz}, 2 \mathrm{H}, \mathrm{CH}), 7.60-7.63(\mathrm{~m}, 3 \mathrm{H}, \mathrm{CH}) .{ }^{13} \mathrm{C} \mathrm{NMR}\left(300 \mathrm{MHz}, \mathrm{CDCl}_{3}\right) ; 16.2,20.1,30.3,85.2,110.9$, 125.5, 127.9, 129.9, 132.8, 137.7, 156.3. IR $\left(\mathrm{KBr}\right.$ pellets $\left.\mathrm{cm}^{-1}\right) \vee 1455,1600,1615,2975,3110$. Anal. CHN: calcd C 77.58, H 7.51, N 6.96, found C 77.70, H 7.40, N 6.99. LC-MS: calcd for $\mathrm{C}_{13} \mathrm{H}_{15} \mathrm{NO}\left[\mathrm{M}^{+}\right]$: 201.12, found 202.02 .

\subsubsection{6-Propyl-3-p-Tolyl-6H-1,2-Oxazine (5q)}

Obtained from $\mathbf{1 b}(0.74 \mathrm{~g}, 5 \mathrm{mmol})$, chloramine-T. $3 \mathrm{H}_{2} \mathrm{O}(1.43 \mathrm{~g}, 5.1 \mathrm{mmol})$ and $\mathbf{4 d}(0.35 \mathrm{~g}, 5.1 \mathrm{mmol})$ as a brown solid, yield $(0.6 \mathrm{~g}, 54.5 \%)$, m.p. $131-132{ }^{\circ} \mathrm{C},{ }^{1} \mathrm{H} \mathrm{NMR}\left(\mathrm{CDCl}_{3}, 300 \mathrm{MHz}\right): \delta 0.94$ (t, $\left.3 \mathrm{H}, \mathrm{CH}_{3}\right), 1.39-1.44$ $\left(\mathrm{m}, 4 \mathrm{H}, \mathrm{CH}_{2}-\mathrm{CH}_{2}\right), 2.35\left(\mathrm{~s}, 3 \mathrm{H}, \mathrm{CH}_{3}\right), 4.18(\mathrm{~m}, 1 \mathrm{H}, \mathrm{C} 6), 6.28$ (dd, $\left.J=4.6 \mathrm{~Hz}, J=10.1,1 \mathrm{H}, \mathrm{C} 5\right), 6.70$ (d, $J=10.2$ $\mathrm{Hz}, 1 \mathrm{H}, \mathrm{CH} 2), 7.20$ (dd, $J=7 \mathrm{~Hz}, J=2 \mathrm{~Hz}, 2 \mathrm{H}, \mathrm{CH}), 7.65$ (dd, $J=7 \mathrm{~Hz}, J=2 \mathrm{~Hz}, 2 \mathrm{H}, \mathrm{CH}) .{ }^{13} \mathrm{C}$ NMR $(300 \mathrm{MHz}$, $\left.\mathrm{CDCl}_{3}\right) ; 16.2,20.4,23.5,30.1,85.9,110.7,127.9,130.3,133.1,143.2,156.4$. IR $\left(\mathrm{KBr}\right.$ pellets cm $\left.{ }^{-1}\right) \vee 1375,1450$, 1600, 1610, 2985, 3105. Anal. CHN: calcd C 78.10, H 7.96, N 6.51, found C 78.30, H 7.84, N 6.64. LC-MS: calcd for $\mathrm{C}_{14} \mathrm{H}_{17} \mathrm{NO}\left[\mathrm{M}^{+}\right]: 215.13$, found 216.05 .

\subsubsection{3-(4-Chlorophenyl)-6-Propyl-6H-1,2-Oxazine (5r)}

Obtained from $1 \mathrm{c}(0.84 \mathrm{~g}, 5 \mathrm{mmol})$, chloramine- $\mathrm{T}^{3} \mathrm{H}_{2} \mathrm{O}(1.43 \mathrm{~g}, 5.1 \mathrm{mmol})$ and $\mathbf{4 d}(0.35 \mathrm{~g}, 5.1 \mathrm{mmol})$ as a colorless solid, yield $(0.65 \mathrm{~g}, 53.7 \%)$, m.p. $131-132{ }^{\circ} \mathrm{C},{ }^{1} \mathrm{H}$ NMR $\left(\mathrm{CDCl}_{3}, 300 \mathrm{MHz}\right): \delta 0.92\left(\mathrm{t}, 3 \mathrm{H}, \mathrm{CH}_{3}\right)$, $1.38-1.43\left(\mathrm{~m}, 4 \mathrm{H}, \mathrm{CH}_{2}-\mathrm{CH}_{2}\right), 4.21(\mathrm{~m}, 1 \mathrm{H}, \mathrm{C} 6), 6.24$ (dd, $\left.J=4.6 \mathrm{~Hz}, J=10.1 \mathrm{~Hz}, 1 \mathrm{H}, \mathrm{C} 5\right), 6.72(\mathrm{~d}, J=10.2 \mathrm{~Hz}$, $1 \mathrm{H}, \mathrm{C} 4), 7.42(\mathrm{~d}, J=8.7 \mathrm{~Hz}, 2 \mathrm{H}, \mathrm{CH}), 7.46(\mathrm{~d}, J=8.7 \mathrm{~Hz}, 2 \mathrm{H}, \mathrm{CH}) .{ }^{13} \mathrm{C}$ NMR $\left(300 \mathrm{MHz}, \mathrm{CDCl}_{3}\right) ; 16.2,20.4$, 30.3, 85.4, 110.9, 127.8, 129.8, 131.2, 133.7, 137.7, 156.5. IR (KBr pellets cm $\left.{ }^{-1}\right) \vee 1440,1600,1615,2965,3112$ Anal. CHN: calcd C 66.24, H 5.99, N 5.94, found C 66.40, H 5.94, N 5.84. LC-MS: calcd for $\mathrm{C}_{13} \mathrm{H}_{14} \mathrm{ClNO}\left[\mathrm{M}^{+}\right]$: 235.08 , found 237.02 .

\subsubsection{3-(4-Methoxyphenyl)-6-Propyl-6H-1,2-Oxazine (5s)}

Obtained from $1 \mathbf{d}(0.82 \mathrm{~g}, 5 \mathrm{mmol})$, chloramine-T. $3 \mathrm{H}_{2} \mathrm{O}(1.43 \mathrm{~g}, 5.1 \mathrm{mmol})$ and $\mathbf{4 d}(0.35 \mathrm{~g}, 5.1 \mathrm{mmol})$ as a colorless solid, yield $(0.7 \mathrm{~g}, 59.3 \%)$, m.p. $131-132{ }^{\circ} \mathrm{C},{ }^{1} \mathrm{H}$ NMR $\left(\mathrm{CDCl}_{3}, 300 \mathrm{MHz}\right): \delta 0.89\left(\mathrm{t}, 3 \mathrm{H}, \mathrm{CH}_{3}\right)$, 1.38-1.44 (m, $\left.4 \mathrm{H}, \mathrm{CH}_{2}-\mathrm{CH}_{2}\right), 3.82\left(\mathrm{~s}, 3 \mathrm{H}, \mathrm{OCH}_{3}\right), 4.20(\mathrm{~m}, 1 \mathrm{H}, \mathrm{C} 6), 6.31(\mathrm{dd}, J=4.6 \mathrm{~Hz}, J=10.1 \mathrm{~Hz}, 1 \mathrm{H}, \mathrm{C} 5)$, $6.72(\mathrm{~d}, J=10.2 \mathrm{~Hz}, 1 \mathrm{H}, \mathrm{C} 4), 7.10(\mathrm{dd}, J=8.7 \mathrm{~Hz}, J=2 \mathrm{~Hz}, 2 \mathrm{H}, \mathrm{CH}), 7.65(\mathrm{dd}, J=6 \mathrm{~Hz}, J=2 \mathrm{~Hz}, 2 \mathrm{H}, \mathrm{CH}){ }^{13} \mathrm{C}$ NMR (300 MHz, $\left.\mathrm{CDCl}_{3}\right) ; 16.1,20.7,30.2,58.6,85.1,110.5,114.4,127.9,130.1,133.1,156.3,160.7$. IR ( $\mathrm{KBr}$ pellets $\left.\mathrm{cm}^{-1}\right) \vee 1240,1445,1600,2955,3040,3075$. Anal. CHN: calcd C 72.70, H 7.41, N 6.06, found C 72.60, 
$\mathrm{H}$ 7.54, N 6.14. LC-MS: calcd for $\mathrm{C}_{14} \mathrm{H}_{17} \mathrm{NO}_{2}\left[\mathrm{M}^{+}\right]: 231.13$, found 232.05.

\subsubsection{3-(4-Nitrophenyl)-6-Propyl-6H-1,2-Oxazine (5t)}

Obtained from 1e $(0.9 \mathrm{~g}, 5 \mathrm{mmol})$, chloramine- $\mathrm{T} .3 \mathrm{H}_{2} \mathrm{O}(1.43 \mathrm{~g}, 5.1 \mathrm{mmol})$ and $\mathbf{4 d}(0.35 \mathrm{~g}, 5.1 \mathrm{mmol})$ as a pale yellow solid, yield $(0.7 \mathrm{~g}, 55.5 \%)$, m.p. $131-132{ }^{\circ} \mathrm{C},{ }^{1} \mathrm{H}$ NMR $\left(\mathrm{CDCl}_{3}, 300 \mathrm{MHz}\right): \delta 0.90\left(\mathrm{t}, 3 \mathrm{H}, \mathrm{CH}_{3}\right), 1.40-1.46$ $\left(\mathrm{m}, 4 \mathrm{H}, \mathrm{CH}_{2}-\mathrm{CH}_{2}\right), 4.22(\mathrm{~m}, 1 \mathrm{H}, \mathrm{C} 6), 6.32(\mathrm{dd}, J=4.6, J=10.1,1 \mathrm{H}, \mathrm{C} 5), 6.70(\mathrm{~d}, J=10.2,1 \mathrm{H}, \mathrm{C} 4), 7.80(\mathrm{~d}, J=$ $8.8 \mathrm{~Hz}, 2 \mathrm{H}, \mathrm{CH}), 8.15(\mathrm{~d}, J=8.8 \mathrm{~Hz}, 2 \mathrm{H}, \mathrm{CH}) .{ }^{13} \mathrm{C} \mathrm{NMR}\left(300 \mathrm{MHz}, \mathrm{CDCl}_{3}\right) ; 16.2,20.4,30.3,85.2,110.8,122.3$, 127.9, 129.8, 138.8, 153.3, 156.4. IR (KBr pellets cm $\left.\mathrm{cm}^{-1}\right) \vee 1435,1520,1600,1615,2985,3035,3075$. Anal. CHN: calcd C 63.40, H 5.73, N 11.38, found C 63.50, H 5.54, N11.44. LC-MS: calcd for $\mathrm{C}_{13} \mathrm{H}_{14} \mathrm{~N}_{2} \mathrm{O}_{3}\left[\mathrm{M}^{+}\right]: 246.10$, found 247.85 .

\section{Results and Discussion}

The compounds 1(a-e) were synthesized by the reaction of ketones with hydroxylamine hydrochloride in the presence of sodium acetate (Brian et al., 2006). Chloramine-T was used as an effective reagent for the generation of $\alpha$-nitrosoolefins from the corresponding ketoximes 1(a-e) (Gaonkar \& Rai, 2005; Manjula et al., 2009), which subsequently underwent hetero Diels-Alder reaction with terminal acetylenes 4(a-d), to give $6 H$-1,2-Oxazine derivatives 5(a-t) in good yield (Table1).

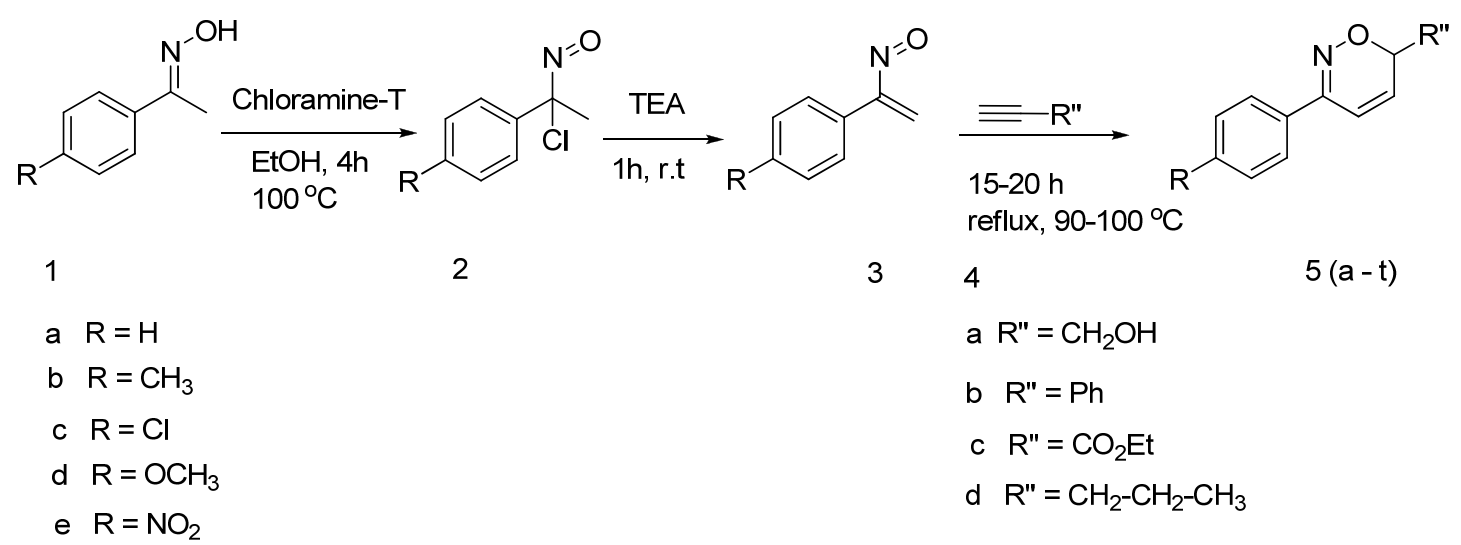

Scheme 1. One-pot synthesis of $6 H$-1,2-oxazine derivatives

Table 1. Structural formulae, time of reaction to convert compounds 3 to 5 and $\%$ yields of the synthesized compounds 5 (a-t)

\begin{tabular}{|c|c|c|c|}
\hline Compound & 6H-1,2-Oxazine & Time $(3 \rightarrow 5) \mathrm{hrs}$ & Yield \% \\
\hline $5 \mathrm{a}$ & & 15 & 64 \\
\hline $5 b$ & & 15 & 60 \\
\hline $5 \mathrm{c}$ & & 17 & 54 \\
\hline $5 \mathrm{~d}$ & & 16 & 64 \\
\hline $5 e$ & & 18 & 60 \\
\hline $5 f$ & & 15 & 51 \\
\hline $5 \mathrm{~g}$ & & 16 & 56 \\
\hline
\end{tabular}




(16)

The identities of compounds (5a-t) were established by their ${ }^{1} \mathrm{H}$ NMR, ${ }^{13} \mathrm{C}$ NMR, IR, elemental analysis and MS. Taking (5a) as an example, the ${ }^{1} \mathrm{H}$ NMR spectrum of (5a) displays broad band at 2.13 due to $\mathrm{OH}$. The $\mathrm{CH}_{2}$ displays multiplets at 3.75-3.93, while the oxazine ring protons appear as a doublet at 6.69 , doublet of doublet at 6.33 and multiplets at $4.20 \mathrm{ppm}$ for the respective protons in the position 4, 5 and 6 . The five protons of phenyl groups appeared as triplets at $\delta 7.40$ with coupling constant $6 \mathrm{~Hz}$ and multiplets at $7.66-7.69 \mathrm{ppm}$. The IR spectra of $6 \mathrm{H}-1,2$-oxazine derivative (5a) showed absorption at $1615 \mathrm{~cm}^{-1}$ owing to the ring nitrogen $(\mathrm{C}=\mathrm{N})$ and 1600 $\mathrm{cm}^{-1}$ due to aromatic ring. The $\mathrm{OH}$ group appears as an absorption band at $3340 \mathrm{~cm}^{-1}$. In the ${ }^{13} \mathrm{C}$ NMR spectra all oxazines gave consistent signals for the newly formed ring carbons. For instance the signals in the region due to $\mathrm{C}_{6}$ appear in the region $\delta 82.4-89.9 \mathrm{ppm}$ while $\mathrm{C}_{5}, \mathrm{C}_{4}$ and $\mathrm{C}_{3}$ appears in the region 90.1, 110.9 and 156.2. The mass spectrum of (5a) showed the molecular ion peak at $\mathrm{m} / \mathrm{z} 189.08$. The formation of the products was further supported by elemental analyses. 1,2-Oxazine can be used as a synthone in the synthesis of $\gamma$-hydroxy ketones, 1,4-diketones etcetera (Sunil \& Rai, 2012).

\section{Conclusions}

In conclusion a new series of $6 H-1,2$-oxazine derivatives were synthesized from ketoximes and terminal alkynes. 
Chloramine-T was used to accomplish this reaction. Yields were moderate to good. Instead of the formation of $4 H-1,2$-Oxazine we observed that this reaction yielded $6 H-1,2$-Oxazine.This was attributed to the formation of a conjugated diene like system.

\section{Acknowledgments}

Authors wish to thank the University of Mysore, India which provided all the facilities that helped in completing this research work.

\section{References}

Brasholz, M., Reissig, H. U., \& Zimmer, R. (2009). Sugars, alkaloids, and heteroaromatics: exploring heterocyclic chemistry with alkoxyallenes. Accounts of Chemical Research, 42(1), 45-56. http://dx.doi.org/10.1021/ar800011h

Brian, S. F., Antony, J. H., Peter, W. G. S., \& Austin, R. T. (2006). Vogel's textbook of practical organic chemistry (5th ed.). Dorling Kindersley (India) Pvt. Ltd.

Buchholz, M., \& Reibig, H. U. (2003). Enantioselective synthesis of the pyrrolidine core of endothelin antagonist ABT- 627 (Atrasentan) via 1,2-Oxazines. European Journal of Organic Chemistry, 2003(18), 3524-3533. http://dx.doi.org/10.1002/ejoc.200300234

Cardona, F., \& Goti, A. (2005). The discovery of novel metal-induced reactions of nitrones: Not only electrophiles and reagents for [3+2] cycloadditions. Angewandte Chemie International Edition, 44(48), 832-7835. http://dx.doi.org/10.1002/anie.200502640

David, E. D., Thomas, L. G., \& Tony G. R. (1983). The cycloaddition of $\alpha$-nitrosostyrenes to olefins. Investigations of the scope and mechanism of the reaction. Journal of the Chemical Society, Perkin Transactions, 1, 1275-1281. http://dx.doi.org/10.1039/p19830001275

Gaonkar, S. L., \& Rai, K. M. L. (2005). A new method for generation of $\alpha$-nitrosolefins from ketoximes and its application to the synthesis of 5,6-dihydro-4H-1,2-oxazines derivatives. Journal of Heterocylic Chemistry, 42, 877-881. http://dx.doi.org/10.1002/jhet.5570420520

Gilchrist, T. L. (1983). Nitroso-alkenes and nitroso-alkynes. Chemical. Society. Reviews., 12, 53-73. http://dx.doi.org/10.1039/cs9831200053

Gilchrist, T. L., \& Wood, J. E. (1996). Comperhensive heterocyclic chemistry II (pp. 279-299, pp. 1177-1307). Oxford, UK: Elsevier. http://dx.doi.org/10.1016/B978-008096518-5.00120-9

Helms, M., Schade, W., Pulz, R., Watanabe, T., Al-Harrasi, A., Fisera, L., ... Reissig, H. U. (2005). Stereodivergent syntheses of highly substituted enantiopure 4-alkoxy-3,6-dihydro-2H-1,2-oxazines by addition of lithiated alkoxyallenes to carbohydrate-derived aldonitrones. European Journal of Organic Chemistry, 2005(6), 1003-1019. http://dx.doi.org/10.1002/ejoc.200400627

Homann, K., Angermann, J., Collas, M., Zimmer, R., \& Reissig, H. U. (1998). Synthesis of 6-ethoxy-6H-1,2-oxazines by hetero Diels-Alder reaction of 1-bromo-2-ethoxyethene with $\alpha$-nitroso alkenes. Journal für Praktische Chemie/Chemiker-Zeitung, 340(7), 649-655. http://dx.doi.org/10.1002/prac.19983400709

Kumarn, S., Shaw, D. M., \& Ley, S. V. (2006). A highly selective, organocatalytic route to chiral 1,2-oxazines from ketones. Chemical Communications, 30, 3211-3213. http://dx.doi.org/10.1039/b606338a

Lu, C. D., \& Zakarian, A. (2008). Total synthesis of $( \pm$ )-trichodermamide $\mathbb{B}$ and of a putative biosynthetic precursor to aspergillazine A using an oxaza-cope rearrangement. Angewandte Chemie International Edition, 47, 6829-6831. http://dx.doi.org/10.1002/ange.200801652

Lyapkalo, I. M., \& Ioffe, S. L. (1998). Conjugated nitrosoalkenes. Russian.Chemical. Reviews (Engl Transl.), 67(6), 467-485. http://dx.doi.org/10.3762/bjoc.5.44

Manjula, M. K., Rai, K. M. L.,Gaongar, S. L., Raveesha, K. A., \& Satish, S. (2009). Synthesis of new series of 5,6-dihydro-4H-1,2-oxazines via hetero Diels-Alder reaction and evaluation of antimicrobial activity. European Journal of Medicinal Chemistry, 44(1), 280-288. http://dx.doi.org/10.1016/j.ejmech.2008.02.027

Mark, S. B., Xiaoming, L., Jumaa, R. Al Dulyymi, Alexander, I. K., \& Valery, A. P. (1993). Unusual chemo- and stereo-selectivities in the reactions of 1,2-dichlorocyclopropenes with nitrile oxides. Journal of Chemical. Society, Perkin Transations, 1(21), 2507-2508. http://dx.doi.org/10.1039/p19930002507

Mori, K., \& Wu, J. (1991). Pheromone synthesis, CXXV. Synthesis of the four possible stereoisomers of 
3,7-dimethylnonadecane, the female sex pheromone of Agromyza frontella Rondani. Liebigs Annalen Chemie, 1991(3), 213-217. http://dx.doi.org/10.1002/jlac.199119910138

Reissig, H. U., Homann, K., Hiller, F., \& Zimmer, R. (2007). A stereoselective access to dihydroxylated pyrrolidines by reductive ring contraction of 1,2-oxazines. Synthesis-stuttgart, 2007(17), 2681-2689. http://dx.doi.org/10.1055/s-2007-983802

Shigeo, T., Hideshi, M., Makoto, H., \& Akio, T. (1991). Synthesis of 6-alkoxy-3-aryl-6-trimethylsilyloxy-5,6-dihydro-4H-1,2-oxazines and their acid-catalysed hydrolysis leading to 3-aryl-5,6-dihydro-4H-1,2-oxazin-6-ones and/or 4-aryl-4-(hydroxyimino)-butyric acid esters. Journal of Chemical. Society, Perkin Transations, 1(12), 3153-3157. http://dx.doi.org/10.1039/p19910003153

Sibi, M. P., Ma, Z., \& Jasperse, C. P. (2005). Enantioselective addition of nitrones to activated cyclopropanes. Journal of the American Chemical Society, 127(16), 5764-5765. http://dx.doi.org/10.1021/ja0421497

Sukhorukov, A. Yu., Lesiv, A. V., Khomutova, Y. A., \& Ioffe, S. L. (2009). Diastereoselective Synthesis of $\gamma$ Amino Acids and Their Derivatives from Nitroethane via Intermediacy of 5,6-Dihydro-4H-1,2-oxazines Bearing the $\mathrm{CH}_{2} \mathrm{CH}\left(\mathrm{CO}_{2} \mathrm{Me}\right)_{2}$ Substituent at C3. Synthesis, 5, 741-754. http://dx.doi.org/10.1055/s-0028-1083360

Sung, C. Y., Kyongtae, K., \& Yunng, J. P. (2001). Hetero-Diels-Alder reactions of $\alpha$-aryl- $\beta$-monohalo- $\alpha$-nitrosoethylenes. diastereoselective synthesis of 6 -substituted 3-Aryl-4-halo-5,6-dihydro-4H-1,2-oxazines. The journal of Organic. Chemistry, 66(22), 7334-7341. http://dx.doi.org/10.1021/jo010476g

Sunil, K. P., \& Rai, K. M. L. (2012). Reductive cleavage of 1,2-oxazines promoted by zinc and ammonium chloride; mild one pot preparation of $\gamma$-hydroxyl ketones. E-Journal of Chemistry, 91(1), 131-136.

Thomas, L. G., Deborah H., Wayne S., \& Ewan, J. T. C. (1987). Formation of 2-acetylpyridines by the base-catalysed ring opening of dihydro- $4 H$-furo[2,3-e] oxazines. Journal of Chemical. Society, Perkin Transations, 1, 2505-2509. http://dx.doi.org/10.1039/p19870002505

Tishkov, A. A., Reissig, H. U., \& Ioffe, S. L. (2002). Preparation of cyclic and bicyclic $\beta$-amino Acids derivatives from methyl 6-ethoxy-5,6-dihydro-4H-1,2-oxazine-4-carboxylate. Synlett, 6, 0863-0866. http://dx.doi.org/10.1055/s-2002-31908

Tsoungas, P. G. (2002). 1,2-Oxazines and their $N$-oxides in synthesis. Heterocycles, 57(5), 915-953. http://dx.doi.org/10.3987/REV-02-548

Young, I. S., \& Kerr, M. A. (2003). A homo [3+2] dipolar cycloaddition: The reaction of nitrones with cyclopropanes. Angewandte Chemie International Edition, 42, 3023-3026. http://dx.doi.org/10.1002/ange.200351573

Zimmer, R., Collas, M., Czerwonka, R., Hain, U., \& Reissig, H. U. (2008). A new access to pyrrolizidine derivatives: Ring contraction of methyl (E)-[1,2-oxazin-3-yl] propenoates. Synthesis, 2, 237-244. http://dx.doi.org/10.1055/s-2007-990946

Zimmer, R., Hoffmann, M., \& Reissig, H. U. (1992c). Model studies of the reduction of 3-phenyl-6H-1,2-oxazines, chemo- and stereoselectivity: synthesis of amino alcohols, amino acids, and related compounds. Chemische Berichte, 125(10), 2243-2248. http://dx.doi.org/10.1002/cber.19921251012

Zimmer, R., Homann, K., \& Reissig, H. U. (1993). Reductive transformations of $6 H$-1,2-oxazines with hydride reagents: Formation of aziridines and 3-hydroxyalkylated-1,2-oxazines. Liebigs Annalen Chemie, 1993(10), 1155-1157. http://dx.doi.org/10.1002/jlac.1993199301186

Zimmer, R., Orschel, B., Scherer, S., \& Reissig, H. U. (2002). Hetero Diels-Alder reactions of nitroso lkenes with alkoxyallene derivatives bearing carbohydrate auxiliaries-asymmetric synthesis of $6 H-1,2$-oxazines and subsequent reductive transformations. Synthesis, 2002(11), 1553-1563.

Zimmer, R., \& Reissig, H. U. (1988). Methoxyallenes as Components in Diels-Alder Reactions with Inverse Electron Demand: Synthesis of 6H-1,2-Oxazines. Angewandte Chemie International Edition, 27(11), 1518-1519. http://dx.doi.org/10.1002/anie.198815181

Zimmer, R., \& Reissig, H. U. (1991). Synthesis of 6H-1,2-oxazines by hetero Diels-Alder reactions of nitroso alkenes towards methoxyallenes. Liebigs Annalen Chemie, 1991(6), 553-562. http://dx.doi.org/10.1002/jlac.1991199101101 
Zimmer, R., \& Reissig, H. U. (1992a). Efficient synthesis of trifluoromethyl-substituted 5,6-dihydro-4H-1,2-oxazines by the hetero-diels-alder reaction of 1,1,1-trifluoro-2-nitroso-2-propene and electron-rich olefins. The Journal of Organic Chemistry, 57(1), 339-347. http://dx.doi.org/10.1021/jo00027a058

Zimmer, R., Reissig, H. U., \& Lindner, H. J. (1992b). Unerwartete bildung von $\beta$-lactamen aus $6 H$-1,2-oxazinen unter lewis-säure-einwirkung. Liebigs Annalen Chemie, 1992(6), 621-624. http://dx.doi.org/10.1002/jlac.1992199201106

Zimmer, R., Schmidt , E., Andra, M., Duhs, M. A., Linder, I., \& Reissig, H. U. (2009). Palladium-catalyzed cross coupling reactions of 4-bromo-6H-1,2-oxazines. Beilstein Journal of Organic Chemistry, 5, 44. http://dx.doi.org/10.3762/bjoc.5.44

\section{Copyrights}

Copyright for this article is retained by the author(s), with first publication rights granted to the journal.

This is an open-access article distributed under the terms and conditions of the Creative Commons Attribution license (http://creativecommons.org/licenses/by/3.0/). 